Document downloaded from:

http://hdl.handle.net/10251/107384

This paper must be cited as:

Gareis, MI.; Lattanzi, M.; Thome, N. (2017). Nilpotent matrices and the minus partial order. Quaestiones Mathematicae. 40(4):519-525. doi:10.2989/16073606.2017.1300612

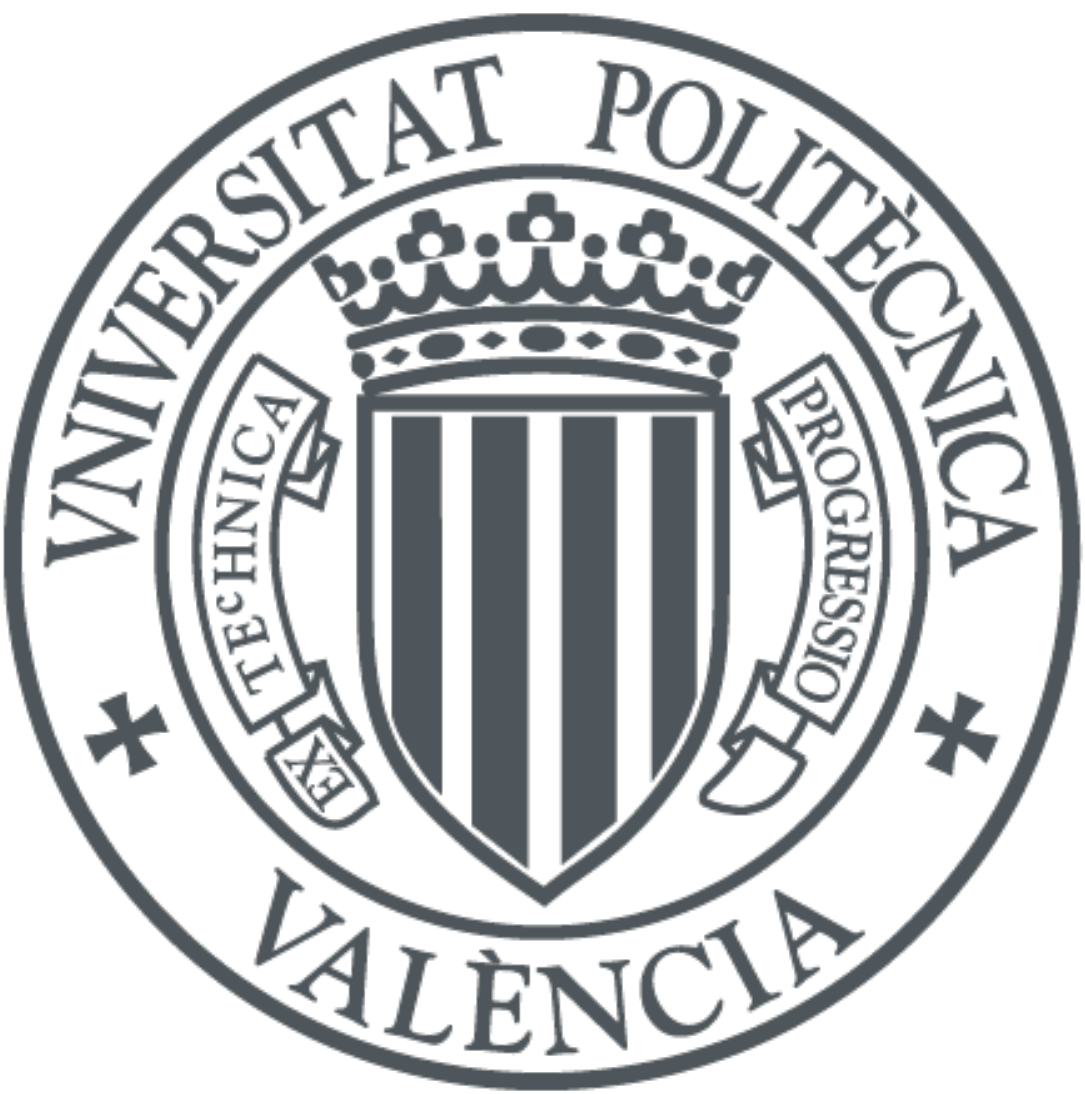

The final publication is available at

https://doi.org/10.2989/16073606.2017.1300612

Copyright Informa UK (National Inquiry Services Center)

Additional Information 


\title{
Nilpotent matrices and the minus partial order*
}

\author{
M.I. Gareis ${ }^{\dagger} \quad$ M. Lattanzi ${ }^{\ddagger} \quad$ N. Thome ${ }^{\S}$
}

\begin{abstract}
In this paper, $\{1\}$-inverses of a nilpotent matrix as well as matrices above a given nilpotent matrix under the minus partial order are characterized.
\end{abstract}

AMS Classification: 15A09, 06A06

Keywords: generalized inverse; minus partial order; nilpotent matrix.

\section{Introduction and background}

Partial orders and preorders on matrices were studied by several authors. They involve different generalized inverses in their definitions. Some results about matrices, generalized inverses and partial orders on matrices can be found, for instance, in [2, 3, 10, 12, 13].

In [10] the Drazin preorder was defined by means of the Drazin inverse, which does not take into account the nilpotent part of the matrices. For this reason, it is not antisymmetric and then Drazin preorder is not a partial order. By considering the nilpotent

*This paper was partially supported by Universidad Nacional de La Pampa, Facultad de Ingeniería (grant Resol. No 155/14). The third author was partially supported by Ministerio de Economía y Competitividad of Spain (Grant number DGI MTM2013-43678-P and Grant Red de Excelencia MTM201568805-REDT).

†Facultad de Ingeniería, Universidad Nacional de La Pampa, General Pico, La Pampa. Argentina. E-mail: marygareis@ing.unlpam.edu.ar.

${ }^{\ddagger}$ Facultad de Ciencias Exactas y Naturales, Universidad Nacional de La Pampa, Av. Uruguay 151, Santa Rosa, La Pampa. Argentina. E-mail: mblatt@exactas.unlpam.edu.ar .

§Instituto Universitario de Matemática Multidisciplinar, Universitat Politècnica de València, 46022, València, Spain. E-mail: njthome@mat.upv.es . 
part of a matrix, a binary relation on square matrices was defined in [10, Definition 4.4.17] in order to extend the Drazin pre-order to a partial order. This relation compares the core part of both matrices under the sharp partial order and the nilpotent part under the minus order. The sharp partial order, defined for index one matrices, has been widely studied [10]. However, to our acknowledge, the minus partial order for nilpotent matrices has not been investigated and it will be partially considered in this paper. In $[8,9]$ similar relations to Drazin preorder were studied for rectangular matrices, and then extended to operators on Banach spaces in $[4,11]$.

Let $\mathbb{C}^{m \times n}$ be the space of complex $m \times n$ matrices and $\mathbb{C}^{n}$ be the complex vector space of $n \times 1$ vectors. The symbols $A^{*}, A^{t}, A^{-1}, \mathcal{R}(A)$, and $\mathcal{N}(A)$ stand for the conjugate transpose, the transpose, the inverse $(m=n)$, the range and the null spaces of a matrix $A \in \mathbb{C}^{m \times n}$, respectively. As usual, $I_{n}$ and $O_{n}$ denote the $n \times n$ identity and zero matrices, respectively. The subscripts will be omitted when no confusion is caused. The index of a matrix $A \in \mathbb{C}^{n \times n}$, denoted by $\operatorname{ind}(A)$, is the smallest nonnegative integer $k$ such that $\mathcal{R}\left(A^{k}\right)=\mathcal{R}\left(A^{k+1}\right)$. A matrix $A \in \mathbb{C}^{n \times n}$ is nilpotent if $A^{r}=O$ for some positive integer $r$, and the smallest such $r$ is called the nilpotence index of $A$ and coincides with $\operatorname{ind}(A)$.

Let $A \in \mathbb{C}^{m \times n}$. A matrix $A^{-} \in \mathbb{C}^{n \times m}$ is a $\{1\}$-inverse of $A$ if $A A^{-} A=A$. A matrix $X \in \mathbb{C}^{n \times m}$ is the Moore-Penrose inverse of $A$ if it satisfies $A X A=A, X A X=X$, $(A X)^{*}=A X$, and $(X A)=(X A)^{*}$. The Moore-Penrose inverse of a matrix $A$ always exists, is unique and it is denoted by $A^{\dagger}$.

On the other hand, the minus partial order, denoted by $\leq^{-}$, was introduced by Hartwig in [5] and it was analyzed by several authors, among others $[1,6,7]$. For two given matrices $A, B \in \mathbb{C}^{m \times n}$, it is said that $A \leq^{-} B$ if and only if $\operatorname{rank}(B-A)=\operatorname{rank}(B)-\operatorname{rank}(A)$. Another equivalent definition for the minus partial order is the following: $A \leq^{-} B$ if and only if there exists a $\{1\}$-inverse $A^{-}$of $A$ such that $A^{-} A=A^{-} B$ and $A A^{-}=B A^{-}$.

For every nonzero nilpotent matrix $N \in \mathbb{C}^{n \times n}$ of index $k$, there exist a block-diagonal matrix $J \in \mathbb{C}^{n \times n}$, called the Jordan canonical form of $N$, and a nonsingular matrix $P \in \mathbb{C}^{n \times n}$ such that

$$
N=P J P^{-1} \quad \text { with } \quad J=\operatorname{diag}\left(J_{1}, J_{2}, \ldots, J_{s}\right)
$$

where $s=\operatorname{dim}(\mathcal{N}(N))$ and the matrix $J_{j}$ is a square matrix of size $n_{j} \times n_{j}$ whose entries are equal to 1 along the superdiagonal and zero elsewhere, called nilpotent Jordan block, for 
each $j \in\{1,2, \ldots, s\}$. Note that if $n_{j}=1$ then $J_{j}=[0]$. When this type of blocks appears, they are located in the lower right corner. If there is more than one, it is considered that $J_{s}$ is the zero matrix of suitable size. If $B$ is a nilpotent Jordan block then $B^{\dagger}=B^{t}$, hence $J^{\dagger}=J^{t}[2]$. The following results are used later.

Theorem 1.1 [2] Let $A \in \mathbb{C}^{m \times n}, B \in \mathbb{C}^{p \times q}$, and $C \in \mathbb{C}^{m \times q}$. The following conditions are equivalent:

(a) The matrix equation $A X B=C$ has a solution.

(b) $A A^{\dagger} C=C$ and $C B^{\dagger} B=C$.

In this case, the set of all the solutions of $A X B=C$ is given by $X=A^{\dagger} C B^{\dagger}+Y-$ $A^{\dagger} A Y B B^{\dagger}$, for arbitrary $Y \in \mathbb{C}^{n \times p}$.

Theorem $1.2[10]$ Let $A, B \in \mathbb{C}^{m \times n}$. Then $A \leq^{-} B$ if and only if

$$
B=A+\left(I_{m}-A A^{-}\right) W\left(I_{n}-A^{-} A\right)
$$

for some matrix $W \in \mathbb{C}^{m \times n}$ and for some $\{1\}$-inverse $A^{-}$of $A$.

\section{$2\{1\}$-inverses of nilpotent matrices}

In this section, $\{1\}$-inverses of a nilpotent matrix are characterized by using its Jordan canonical form. Notice that for the nilpotent matrix $N=O$, every matrix $N^{-}$satisfies $O N^{-} O=O$, so only nonzero nilpotent matrices will be considered.

Theorem 2.1 Let $N \in \mathbb{C}^{n \times n}$ be a nonzero nilpotent matrix written in its Jordan canonical form $N=P J P^{-1}$ as in (1). A matrix $N^{-} \in \mathbb{C}^{n \times n}$ is $\{1\}$-inverse of $N$ if and only if $N^{-}=P X P^{-1}$, where the partition of $X=\left[X_{i j}\right]_{1 \leq i, j \leq s}$ has been carried out according to the size of the blocks of $J$ and

$$
X_{i j}=\left[\begin{array}{cc}
\left(u^{(i j)}\right)^{t} & \alpha^{(i j)} \\
R & v^{(i j)}
\end{array}\right]
$$

where $u^{(i j)} \in \mathbb{C}^{n_{j}-1}, v^{(i j)} \in \mathbb{C}^{n_{i}-1}$, and $\alpha^{(i j)} \in \mathbb{C}, R \in \mathbb{C}^{\left(n_{i}-1\right) \times\left(n_{j}-1\right)}$ is either the identity matrix (for $i=j$ ) or the zero matrix (for $i \neq j$ ), for all $1 \leq i, j \leq s$. 
Proof. Let $N \in \mathbb{C}^{n \times n}$ be a nonzero nilpotent matrix such that $N=P J P^{-1}$ where $P \in \mathbb{C}^{n \times n}$ is nonsingular, $J=P^{-1} N P=\operatorname{diag}\left(J_{1}, J_{2}, \ldots, J_{s}\right), s=\operatorname{dim}(\mathcal{N}(N))$, and $J_{j}$ is a nilpotent Jordan block of size $n_{j} \times n_{j}$, for each $j \in\{1,2, \ldots, s\}$. It is clear that $N^{-} \in \mathbb{C}^{n \times n}$ is a $\{1\}$-inverse of $N$ if and only if $X=P^{-1} N^{-} P$ is a $\{1\}$-inverse of $J$.

Let $X=\left[X_{i j}\right]_{1 \leq i, j \leq s}$ be a partition of $X$ according to the blocks of $J$; i.e., each block $X_{i j}$ has order $n_{i} \times n_{j}$. By making the products, it then follows $J X J=\left[J_{i} X_{i j} J_{j}\right]_{1 \leq i, j \leq s}$ thus, $X$ is a $\{1\}$-inverse of $J$ if and only if

$$
\begin{gathered}
J_{i} X_{i i} J_{i}=J_{i}, \quad i \in\{1, \ldots, s\} \\
J_{i} X_{i j} J_{j}=O, \quad i, j \in\{1, \ldots, s\}, \quad i \neq j .
\end{gathered}
$$

In order to solve matrix equations (2a), Theorem 1.1 is applied by setting $A=B=$ $C=J_{i}$, for $i \in\{1, \ldots, s\},\left(J_{i}^{\dagger}=J_{i}^{t}\right)$. Hence, the general solution of (2a) is given by

$$
X_{i i}=J_{i}^{t} J_{i} J_{i}^{t}+Z_{i}-J_{i}^{t} J_{i} Z_{i} J_{i} J_{i}^{t}
$$

where $Z_{i} \in \mathbb{C}^{n_{i} \times n_{i}}$ is arbitrary. It is easy to see that

$$
J_{i}^{t} J_{i}=I_{n_{i}}-e_{1} e_{1}^{t} \quad \text { and } \quad J_{i} J_{i}^{t}=I_{n_{i}}-e_{n_{i}} e_{n_{i}}^{t}
$$

where $e_{1}$ and $e_{n_{i}}$ are the column canonical vectors of $\mathbb{C}^{n_{i}}$. By replacing in (3) the expressions for $J_{i}^{t} J_{i}$ and $J_{i} J_{i}^{t}$ obtained in (4), by making some computations, and using some properties of Moore-Penrose inverse, it is obtained

$$
X_{i i}=J_{i}^{t}+Z_{i} e_{n_{i}} e_{n_{i}}^{t}+e_{1} e_{1}^{t} Z_{i}-e_{1} e_{1}^{t} Z_{i} e_{n_{i}} e_{n_{i}}^{t}
$$

Let us denote the entries of matrices $Z_{i}$ by $z_{h k}^{(i)}$. Then

$$
Z_{i} e_{n_{i}} e_{n_{i}}^{t}=\left[\begin{array}{cccc}
0 & 0 & \cdots & z_{1 n_{i}}^{(i)} \\
0 & 0 & \cdots & z_{2 n_{i}}^{(i)} \\
\vdots & \vdots & \ddots & \vdots \\
0 & 0 & \cdots & z_{n_{i} n_{i}}^{(i)}
\end{array}\right] \quad \text { and } \quad e_{1} e_{1}^{t} Z_{i}=\left[\begin{array}{cccc}
z_{11}^{(i)} & z_{12}^{(i)} & \cdots & z_{1 n_{i}}^{(i)} \\
0 & 0 & \cdots & 0 \\
\vdots & \vdots & \ddots & \vdots \\
0 & 0 & \cdots & 0
\end{array}\right]
$$


Hence, by replacing in expression (5) we obtain

$$
X_{i i}=\left[\begin{array}{cccccc}
z_{11}^{(i)} & z_{12}^{(i)} & z_{13}^{(i)} & \ldots & z_{1 n_{i-1}}^{(i)} & z_{1 n_{i}}^{(i)} \\
1 & 0 & 0 & \ldots & 0 & z_{2 n_{i}}^{(i)} \\
0 & 1 & 0 & \ldots & 0 & z_{3 n_{i}}^{(i)} \\
\vdots & \vdots & \vdots & \ddots & \vdots & \vdots \\
0 & 0 & 0 & \ldots & 0 & z_{n_{i-1} n_{i}}^{(i)} \\
0 & 0 & 0 & \ldots & 1 & z_{n_{i} n_{i}}^{(i)}
\end{array}\right]
$$

In order to solve matrix equations (2b), Theorem 1.1 is applied by setting $A=J_{i}$, $B=J_{j}$, and $C=O$ for $i, j \in\{1, \ldots, s\}\left(J_{k}^{\dagger}=J_{k}^{t}\right.$, for $\left.k \in\{i, j\}\right)$. The general solution of $(2 \mathrm{~b})$ is

$$
X_{i j}=W_{i j}-J_{i}^{t} J_{i} W_{i j} J_{j} J_{j}^{t}
$$

for arbitrary $W_{i j} \in \mathbb{C}^{n_{i} \times n_{j}}$. By replacing (4) in (7) it is obtained

$$
X_{i j}=W_{i j} e_{n_{j}} e_{n_{j}}^{t}+e_{1} e_{1}^{t} W_{i j}-e_{1} e_{1}^{t} W_{i j} e_{n_{j}} e_{n_{j}}^{t} .
$$

Let $W_{i j}=\left[w_{h k}^{(i j)}\right]_{1 \leq h \leq n_{i}, 1 \leq k \leq n_{j}}$. By making the multiplications in (8), it results

$$
X_{i j}=\left[\begin{array}{cccc}
w_{11}^{(i j)} & w_{12}^{(i j)} & \ldots & w_{1 n_{j}}^{(i j)} \\
0 & 0 & \ldots & w_{2 n_{j}}^{(i j)} \\
\vdots & \vdots & \ddots & \vdots \\
0 & 0 & \ldots & w_{n_{i} n_{j}}^{(i j)}
\end{array}\right]
$$

Both situations (6) and (9) can be written simultaneously as follows:

$$
X_{i j}=\left[\begin{array}{cc}
\left(u^{(i j)}\right)^{t} & \alpha^{(i j)} \\
R & v^{(i j)}
\end{array}\right],
$$

where $u^{(i j)} \in \mathbb{C}^{n_{j}-1}, v^{(i j)} \in \mathbb{C}^{n_{i}-1}, \alpha^{(i j)} \in \mathbb{C}$, and $R \in \mathbb{C}^{\left(n_{i}-1\right) \times\left(n_{j}-1\right)}$, for $1 \leq i, j \leq s$, such that

$$
\begin{aligned}
& \left(u^{(i j)}\right)^{t}=\left[\begin{array}{llll}
\beta_{11}^{(i j)} & \beta_{12}^{(i j)} & \cdots & \beta_{1 n_{j-1}}^{(i j)}
\end{array}\right] \quad \text { with } \quad \beta_{1 k}^{(i j)}=\left\{\begin{array}{cc}
z_{1 k}^{(i)} & \text { if } i=j \\
w_{1 k}^{(i j)} & \text { if } i \neq j
\end{array} \quad \text { for } 1 \leq k \leq n_{j}-1,\right. \\
& \left(v^{(i j)}\right)^{t}=\left[\begin{array}{llll}
\gamma_{2 n_{j}}^{(i j)} & \gamma_{3 n_{j}}^{(i j)} & \cdots & \gamma_{n_{i} n_{j}}^{(i j)}
\end{array}\right] \quad \text { with } \quad \gamma_{h n_{j}}^{(i j)}=\left\{\begin{array}{rl}
z_{h n_{i}}^{(i)} & \text { if } i=j \\
w_{h n_{j}}^{(i j)} & \text { if } i \neq j
\end{array}, \text { for } 2 \leq h \leq n_{i},\right.
\end{aligned}
$$




$$
\alpha^{(i j)}=\left\{\begin{array}{cl}
z_{1 n_{i}}^{(i)} & \text { if } i=j \\
w_{1 n_{j}}^{(i j)} & \text { if } i \neq j
\end{array} \text { and } \quad R=\left\{\begin{array}{cc}
I_{\left(n_{i}-1\right) \times\left(n_{i}-1\right)} & \text { if } i=j \\
O_{\left(n_{i}-1\right) \times\left(n_{j}-1\right)} & \text { if } i \neq j
\end{array} .\right.\right.
$$

This completes the proof.

\section{Nilpotent matrices and the minus partial order}

In this section, a characterization for matrices above a given nilpotent matrix under the minus partial order is obtained. It is based on the form of $\{1\}$-inverses of a nilpotent matrix as obtained in Theorem 2.1. Notice that if $N=O$ then every matrix $Y$ with the same size as $N$ satisfies $N \leq^{-} Y$.

Theorem 3.1 Let $N \in \mathbb{C}^{n \times n}$ be a nonzero nilpotent matrix written in its Jordan canonical form as in (1). The following conditions are equivalent:

(a) There exists a matrix $Y \in \mathbb{C}^{n \times n}$ such that $N \leq^{-} Y$,

(b) $Y=N+P V P^{-1} W P U P^{-1}$, for some $W \in \mathbb{C}^{n \times n}$, and $V, U \in \mathbb{C}^{n \times n}$ partitioned according to the size of the blocks of $J, V=\left[V_{i j}\right]_{1 \leq i, j \leq s}, U=\left[U_{i j}\right]_{1 \leq i, j \leq s}$,

$$
\begin{gathered}
V_{i j}=\left[\begin{array}{cc}
O & v^{(i j)} \\
0^{t} & \delta_{i j}
\end{array}\right]_{n_{i} \times n_{j}}, \quad U_{i j}=\left[\begin{array}{cc}
\delta_{i j} & \left(u^{(i j)}\right)^{t} \\
0 & O
\end{array}\right]_{n_{i} \times n_{j}}, \quad \delta_{i j}=\left\{\begin{array}{cc}
1 & \text { if } i=j \\
0 & \text { if } i \neq j
\end{array},\right. \\
v^{(i j)} \in \mathbb{C}^{n_{i}-1}, \text { and } u^{(i j)} \in \mathbb{C}^{n_{j}-1}, \text { for all } i, j \in\{1,2, \ldots, s\} .
\end{gathered}
$$

Proof. $(a) \Longrightarrow(b)$ Let $N \neq O$ be a nilpotent matrix written as in (1) such that $N \leq^{-} Y$ for some matrix $Y$. Then, by Theorem 1.2,

$$
Y=N+\left(I-N N^{-}\right) W\left(I-N^{-} N\right)
$$

for some matrix $W$ and for some $\{1\}$-inverse $N^{-}$of $N$. By Theorem 2.1, a $\{1\}$-inverse of $N$ can be written as $N^{-}=P X P^{-1}$, where $X$ is partitioned according to the size of the blocks of $J$ such that each block of $X$ has the form

$$
X_{i j}=\left[\begin{array}{cc}
\left(u^{(i j)}\right)^{t} & \alpha^{(i j)} \\
R & v^{(i j)}
\end{array}\right]
$$


where $u^{(i j)} \in \mathbb{C}^{n_{j}-1}, v^{(i j)} \in \mathbb{C}^{n_{i}-1}$ and $\alpha^{(i j)} \in \mathbb{C}$, and $R \in \mathbb{C}^{\left(n_{i}-1\right) \times\left(n_{j}-1\right)}$ is either the identity matrix (for $i=j$ ) or the zero matrix (for $i \neq j$ ), for all $i, j \in\{1,2, \ldots, s\}$.

Post-multiplying (10) by the nonsingular matrix $P$, pre-multiplying it by $P^{-1}$, and taking into account that $N N^{-}=P J X P^{-1}$ and $N^{-} N=P X J P^{-1}$, then equality (10) can be written equivalently as

$$
P^{-1} Y P=J+(I-J X) P^{-1} W P(I-X J) .
$$

It is easy to see that $[J X]_{i j}=J_{i} X_{i j}$ and $[X J]_{i j}=X_{i j} J_{j}$. Let us write $J_{i}=\left[\begin{array}{cc}O & I_{n_{i}-1} \\ 0 & O^{t}\end{array}\right]$ according to the blocks of $X_{i j}$, where $0 \in \mathbb{C}, I \in \mathbb{C}^{\left(n_{i}-1\right) \times\left(n_{i}-1\right)}$ and $O \in \mathbb{C}^{n_{i}-1}$. Then

$$
J_{i} X_{i j}=\left[\begin{array}{cc}
R & v^{(i j)} \\
O^{t} & 0
\end{array}\right] \quad \text { and } \quad X_{i j} J_{j}=\left[\begin{array}{cc}
0 & \left(u^{(i j)}\right)^{t} \\
O & R
\end{array}\right]
$$

with $O \in \mathbb{C}^{n_{j}-1}, 0 \in \mathbb{C}, u^{(i j)} \in \mathbb{C}^{n_{j}-1}, v^{(i j)} \in \mathbb{C}^{n_{i}-1}, R=I_{n_{i}-1}$ if $i=j$, and $R=$ $O_{\left(n_{i}-1\right) \times\left(n_{j}-1\right)}$ if $i \neq j$. Some computations give

$[I-J X]_{i j}=\left[\begin{array}{cc}O & -v^{(i j)} \\ O^{t} & \delta_{i j}\end{array}\right]$ and $[I-X J]_{i j}=\left[\begin{array}{cc}\delta_{i j} & -\left(u^{(i j)}\right)^{t} \\ O & O\end{array}\right]$, where $\delta_{i j}=\left\{\begin{array}{cc}1 & \text { if } i=j \\ 0 & \text { if } i \neq j\end{array}\right.$.

Set $V_{i j}=[I-J X]_{i j}, U_{i j}=[I-X J]_{i j}, V=\left[V_{i j}\right]_{1 \leq i j \leq s}$, and $U=\left[U_{i j}\right]_{1 \leq i j \leq s}$. Hence, from (11), it is clear that $Y=N+P V P^{-1} W P U P^{-1}$, for some matrix $W$.

$(b) \Longrightarrow(a)$ It is an easy computation.

\section{References}

[1] R.B. BApat, S.K. Jain, L. E. SNyder, Nonnegative idempotent matrices and the minus partial order, Linear Algebra and its Applications, 261 (1997), pp. 143-154.

[2] A. Ben-Israel, T. Greville, Generalized Inverses: Theory and Applications. Wiley, $2^{\text {nd }}$ ed., 2003.

[3] S.L. Campbell, C.D. Meyer JR., Generalized Inverses of Linear Transformations. Dover, $2^{\text {nd }}$ ed., 1991. 
[4] D.S. Cvetković-Ilić, D. Mosić, Y. Wei, Partial Orders on B $(H)$, Linear Algebra and its Applications, 481 (15) (2015), pp. 115-130.

[5] R.E. Hartwig, How to partially order regular elements?, Math. Japon., 25 (1980), pp. $1-13$.

[6] R.E. Hartwig, G.P.H. Styan, On some characterizations of the star partial ordering for matrices and rank sustractivity, Linear Algebra and its Applications, 82 (1986) pp. 145-161.

[7] J. Hauke, A. Markiewicz, T. Szulc, Inter-and extrapolary properties of matrix partial orderings, Linear Algebra and its Applications, 332-334 (2001), pp. 437-445.

[8] A. Hernández, M. Lattanzi, N. Thome, Weighted binary relations involving the Drazin inverse, Applied Mathematics and Computation, 253 (2015) pp. 215-223.

[9] A. Hernández, M. Lattanzi, N. Thome, On some new pre-orders defined by weighted Drazin inverses, Applied Mathematics and Computation, 282 (2016) pp. 108-116.

[10] S.K. Mitra, P. Bhimasankaram, S.B. Malik, Matrix partial orders, shorted operators and applications. World Scientific Publishing Company, 2010.

[11] D. Mosić, Weighted binary relations for operators on Banach spaces, to appear in Aequationes Mathematicae, DOI: 10.1007/s00010-016-0413-3.

[12] C. R. RAO, S.K. Mitra, Generalized inverse of matrices and its applications. Wiley, New York, 1971.

[13] G. Wang, Y. Wei, S. QiaO, Generalized Inverses: Theory and Computations, Science Press, Beijing, 2004. 